\title{
Circulating Neutrophil-Derived Microparticles Associated with the Prognosis of Patients with Sepsis
}

This article was published in the following Dove Press journal:

Journal of Inflammation Research

\author{
Hong-Peng Chen ${ }^{1,2, *}$ \\ Xiao-Yan Wang ${ }^{l} *$ \\ Xiao-Yan Pan' \\ Wang-Wang Hu' \\ Shu-Ting Cai ${ }^{1}$ \\ Kiran Joshi ${ }^{3}$ \\ Lie-Hua Deng ${ }^{1,2}$ \\ Daqing $\mathrm{Ma}\left(\mathbb{D}^{3}\right.$
}

'Department of Critical Care Medicine, Affiliated Hospital of Guangdong Medical University, Zhanjiang, Guangdong, People's Republic of China; ${ }^{2}$ Zhanjiang Key Laboratory of Organ Injury and Protection and Translational Medicine, Zhanjiang, Guangdong, People's Republic of China; ${ }^{3}$ Division of Anesthetics, Pain Medicine and Intensive Care, Department of Surgery and Cancer, Faculty of Medicine, Imperial College London, Chelsea and Westminster Hospital, London, UK

*These authors contributed equally to this work

\footnotetext{
Correspondence: Lie-Hua Deng; Daqing $\mathrm{Ma}$

Department of Critical Care Medicine, Affiliated Hospital of Guangdong Medical University, Zhanjiang, Guangdong, China Email glinson@126.com; d.ma@imperial. ac.uk
}

\begin{abstract}
Introduction: Because of its high morbidity and mortality, sepsis remains the leading cause of death in the ICU. Microparticles (MP) have been largely studied as potential diagnostic or prognostic markers in various diseases including sepsis.

Objective: The biological and clinical relevance of neutrophil-derived microparticles (NDMPs) within the MP population remains unclear. The objective of this study was to elucidate the relationship between plasma NDMPs and the prognosis of patients with sepsis and/or septic shock.
\end{abstract}

Methods: The study was designed as an observational, noninterventional clinical study. The cohort for this study included 40 sepsis and 40 septic shock patients together with 10 healthy controls admitted to the Intensive Care Unit (ICU) and the Health Surveillance Center in the Affiliated Hospital of Guangdong Medical University, Zhanjiang, Guangdong, China, from January to November 2018, respectively. The degree of critical disease for sepsis and septic shock was evaluated, with data analyses conducted from 2018 to 2019.

Results: On days 1,3 and 5 post-admission a series of data including plasma NDMP levels, patient demographics, TNF- $\alpha$ levels, IL-6 levels, sTREM-1 levels, and the sepsis severity score measurements were collected. A survival curve was plotted against levels of plasma NDMPs. Levels of NDMPs were observed to be higher in the septic shock patients than in the sepsis patients on days 1,3 , and 5 post-ICU admission $(p<0.05)$. NDMP levels were significantly increased in sepsis and septic shock patients with a parallel increase in pro-inflammatory mediators and sepsis severity score $(p<0.05)$ as well as mortality.

Conclusion: Our data suggest that NDMPs may be a biomarker of sepsis severity and mortality although its implications on sepsis prognosis warrant further study.

Keywords: sepsis, septic shock, cell-derived microparticles, biomarker, prognosis

\section{Introduction}

Extracellular vesicles (EVs) including membrane-derived exosomes, ectosomes, microvesicles, shedding microvesicles, and microparticles (MPs) have considerable biological functions and can mediate intercellular communication. ${ }^{1,2}$ Neutrophils are the first-line immune cells infiltrating into inflamed tissues during acute inflammation and they subsequently produce microparticles (NDMPs) in response to various inflammatory stimuli. NDMPs (100-1000 $\mathrm{nm}$ in diameter) are composed of proteins, lipids and nucleic acids released from activated or damaged neutrophils. ${ }^{3-6}$ NDMPs in body fluids can modulate homeostasis and participate in various pathophysiological processes such as host defense suppression, ${ }^{7,8}$ submit your manuscript DovePress

http://c 
pro-inflammatory effect, ${ }^{9,10}$ and delayed wound healing. ${ }^{11}$ NDMPs have been implicated in a variety of disease processes including host defense, ${ }^{7}$ inflammatory responses, ${ }^{8}$ wound healing, lung injury, cancer and sepsis. ${ }^{12,13}$ CD15 can serve as a specific cell surface protein marker of NDMPs. ${ }^{14}$

Sepsis is characterized by a complex disorder that develops as a dysregulated host response to an infection and is associated with acute organ dysfunction and impaired immune response, ${ }^{15}$ potentially resulting in multiple organ dysfunction syndrome (MODS), multiple organ failure (MOF), ${ }^{16}$ or even death. ${ }^{17}$ Immune cells such as neutrophils, macrophages, dendritic cells (DC), $\mathrm{T}$ lymphocytes, regulatory $\mathrm{T}$ cells (Tregs), and natural killer T (NKT) cells can initiate or suppress inflammation by producing pro-inflammatory cytokines [tumor necrosis factor (TNF)- $\alpha$, interleukin (IL)-6] or antiinflammatory cytokines (eg IL-10) in septic patients. ${ }^{15-19}$ It has been reported that blood biomarkers including soluble triggering receptor expressed on myeloid cells-1 (sTREM-1), procalcitonin (PCT) ${ }^{20}$ and presepsin $(\mathrm{SCD} 14)^{21}$ can be used to evaluate sepsis severity and prognosis, but their feasibility is not optimal yet. ${ }^{22}$ NDMPs may play a role in the development and progression of sepsis, ${ }^{23}$ but their predictive value for septic outcome remains unknown. We hypothesize that sepsis can induce the production of NDMPs, leading to leukocyte inhibition, immunosuppression and poor prognosis. The aims of our study were to describe the levels of NDMPs over the course of sepsis and septic shock and to identity whether levels of NDMPs were related to the severity of illness (based on Acute Physiology and Chronic Health Evaluation II [APACHE II] Score, multiple organ dysfunction syndrome [MODS], mechanical ventilation time, ICU length of stay [LOS] and total hospital length of stay [LOS]). The plasma concentration of NDMPs and pro-inflammatory mediators (sTREM-1, TNF- $\alpha$ and IL-6) was measured in sepsis patients, and their correlation with sepsis severity and mortality was analyzed.

\section{Materials and Methods}

\section{Patients' Enrollment}

A sequential sample of 40 sepsis and 40 septic shock patients together with 10 healthy controls were studied. They were prospectively enrolled from the Intensive Care Unit (ICU) and the Health Surveillance Center at the Affiliated Hospital of Guangdong Medical University, Zhanjiang, Guangdong, China, from January to November 2018, respectively. Patients were followed for 28 days or until death and for purpose of analysis were grouped as survivors and non-survivors according to the 28-day all-cause mortality. Relevant demographic, clinical, and laboratory characteristics were recorded. Demographic data including age, sex, primary disease, medical history, laboratory measurements, mechanical ventilation time, ICU and total length of hospital stay (LOS), and 28-day survival were recorded; in addition to APACHE II and MODS scores that were calculated for each patient. Patients were enrolled within 24-48 hours of the first sepsis-induced organ failure, and samples were taken on day 1, 3, 5 and 7 following the enrollment.

\section{Ethics Statement}

The study protocol was approved by the Ethics Committee of the Affiliated Hospital of Guangdong Medical University, Zhanjiang (2016-093KT), and written informed consent was obtained from patients or their caring relatives.

\section{Inclusion Criteria}

Sepsis was diagnosed and classified using international guidelines for sepsis and septic shock. ${ }^{24}$ Sepsis was defined as an increase in the Sequential (Sepsisrelated) Organ Failure Assessment (SOFA) score of 2 points or more, which was caused by presumed or confirmed infection (Sepsis-3). ${ }^{25}$ In order to confirm the accuracy of included patients, the septic shock cases had to meet all three of the following criteria (1) diagnosis of septic shock based on ICD-10 code of discharge diagnosis, (2) criteria of sepsis described by sepsis-3, and (3) mean arterial pressure (MAP) $<65 \mathrm{mmHg}$ with vasopressor use and serum lactate level $>2 \mathrm{mmol} / \mathrm{l}$ along with an antibiotic prescription. Patients with systemic inflammatory response syndrome were classified as having sepsis if they had a suspected or proven microbial source.

\section{Exclusion Criteria}

Patients were excluded if they had a malignant tumor, immunosuppression or poisoning. Additionally, patients with organ transplantation, neutropenia, and hematologic malignancies were excluded. 


\section{Blood Sampling and Handling}

Peripheral blood $(10 \mathrm{~mL})$ sampling was performed immediately after patients fulfilled sepsis and septic shock criteria and subsequently repeated on days $1,3,5$, and 7 . Samples for NDMPs and ELISA analysis were collected from a non-heparinized arterial line directly into tubes containing EDTA dipotassium salt as an anticoagulant. Platelet-free plasma was prepared directly in our department within one hour of blood collection by centrifugation (10 minutes at $3000 \times \mathrm{g})$ at room temperature. Aliquots were immediately frozen and then stored at $-80^{\circ} \mathrm{C}$ until use. Upon analysis, aliquots of platelet-free plasma were thawed at $37^{\circ} \mathrm{C}$ in a water bath for 2 minutes and then kept on wet ice.

\section{Isolation of NDMPs Using Anti-CDI5 Conjugated Microbeads}

The isolation of MVs was performed as previously reported. ${ }^{26}$ In brief, plasma samples were centrifuged at $300 \mathrm{~g}$ at $4^{\circ} \mathrm{C}$ for $15 \mathrm{~min}$ and followed by centrifugation at $2000 \mathrm{~g}$ at $4^{\circ} \mathrm{C}$ for $30 \mathrm{~min}$ to remove cellular debris. The supernatants were centrifuged at $20000 \mathrm{~g}$ at $4^{\circ} \mathrm{C}$ for 70 mins to pellet the microparticles (MPs). The pelleted MPs were then incubated with $10 \mu \mathrm{L}$ of biotinconjugated anti-CD15 antibody (Milteny Biotech, Bergisch-Gladbach, Germany) in a $100 \mu \mathrm{L}$ reaction volume for $2 \mathrm{hrs}$, followed by adding $10 \mu \mathrm{L}$ of antibiotin microbeads (Milteny Biotech) for $15 \mathrm{~min}$. Then the microbead-labeled MPs from the total MPs suspension were separated using a DynaMag-2 magnet (Life technology, Shanghai, China). After an overnight magnet separation, the fluid was gently removed and the microbead-bound MPs were resuspended with $100 \mu \mathrm{L}$ particle-free PBS which was filtered through a $20 \mathrm{~nm}$ membrane filter (Anotop 25, Whatman, United Kingdom). The multi-sort release reagent (Milteny Biotech) $(10 \mu \mathrm{L})$ was added to cleave off the microbeads. After $10 \mathrm{~min}$, the sample was brought up to a 250 $\mu \mathrm{L}$ final volume with filtered PBS, and placed on the magnet. On the next day, the MPs in the fluid were collected to be CD15+ MPs. All isolated MPs were enumerated using the NTA NS300 system (Malvern, United Kingdom).

\section{Nanoparticle Tracking Analysis}

The NanoSight NS300 (Malvern, United Kingdom) was used to detect MPs. ${ }^{27}$ The NanoSight polystyrene latex calibration beads, $100 \mathrm{~nm}$ and $1000 \mathrm{~nm}$, were used to check the instrument performance. The camera level was maintained at 10 for light scatter mode. Data were analyzed by NTA 3.0 software (Malvern, United Kingdom).

\section{ELISA Assay}

Plasma levels of sTREM-1 (RayBiotech, Norcross, GA, USA), TNF- $\alpha$ and IL-6 (NeoBioscience, Wuhan, China) were determined by ELISA kits according to the manufacturer's protocol.

\section{Statistical Analysis}

Measurement data were expressed as means \pm standard deviation, dot plot or median and range. Statistical analysis was carried out with GraphPad Prism 5.0 (San Diego, CA, USA) and SPSS 22.0 (SPSS, Chicago, IL, USA). A p-value of $<0.05$ was considered to be statistically significant. Univariate analysis was performed using $t$ test, $x^{2}$ test, Spearman correlation, and Wilcoxon rank sum test. Cumulative-survival curves were estimated with the Kaplan-Meier method. Receiver operating characteristic (ROC) curves of NDMPs were constructed.

\section{Results}

Ninety-four patients were screened, of whom 14 were excluded because of immunosuppressant usage (11 cases) and malignant tumors (3 cases). Eighty eligible patients were classified as sepsis $(\mathrm{n}=40)$ and septic shock patients $(n=40)$, and their baseline characteristics at ICU admission are listed in Table 1. Compared to the sepsis patients, the WBC count, APACHE II score and MODS score were significantly higher in the septic shock patients $(p<0.05)$.

\section{Nanoparticle Tracking Analysis of the Plasma NDMPs}

The NDMPs can be easily seen after immunostaining (Figure 1A). The plasma NDMPs were detected in the healthy controls, sepsis and septic shock patients (Figure 1B-D) by Nanoparticle tracking analysis. The plasma concentration of NDMPs in the sepsis and septic shock patients at ICU admission were significantly higher than those in healthy individuals $(p<0.05)$ but they were even higher in the septic shock patients (Figure 1E). 
Table I The Baseline Characteristics of Sepsis and Septic Shock Patients at the ICU Admission

\begin{tabular}{|c|c|c|c|}
\hline Characteristics & $\begin{array}{l}\text { Sepsis } \\
\text { Group } \\
(n=40)\end{array}$ & $\begin{array}{l}\text { Septic } \\
\text { Shock } \\
\text { Group } \\
(n=40)\end{array}$ & $P$ value \\
\hline Age (years) & $65 \pm 15$ & $69 \pm 18$ & Ns \\
\hline Male/female & $25 / 15$ & $30 / 10$ & Ns \\
\hline Temperature $\left({ }^{\circ} \mathrm{C}\right)$ & $38.2 \pm 1.2$ & $38.9 \pm 0.9$ & Ns \\
\hline WBC counts $\left(\times 10^{9} / \mathrm{L}\right)$ & $13.5 \pm 6.2$ & $16.2 \pm 7.9$ & $<0.01$ \\
\hline APACHE II score & $14.7 \pm 0.8$ & $17.8 \pm 0.7$ & 0.0085 \\
\hline MODS score & $5.8 \pm 0.4$ & $7.3 \pm 0.3$ & 0.0072 \\
\hline \multicolumn{4}{|l|}{ Source of infection $(n, \%)$} \\
\hline Lung & $32(80 \%)$ & 34 (85\%) & Ns \\
\hline Abdomen & 15 (37.5\%) & $12(30 \%)$ & Ns \\
\hline Blood stream & $21(52.5 \%)$ & 23 (57.5\%) & Ns \\
\hline Invasive vessel & $31(77.5 \%)$ & $40(100 \%)$ & Ns \\
\hline Skin and soft tissue & $9(22.5 \%)$ & $12(30 \%)$ & Ns \\
\hline Other & $3(7.5 \%)$ & $5(\mid 2.5 \%)$ & Ns \\
\hline \multicolumn{4}{|l|}{ Comorbidities } \\
\hline Current smoker & $19(47.5 \%)$ & $23(57.5 \%)$ & Ns \\
\hline Diabetes & $12(30 \%)$ & $17(47.5 \%)$ & Ns \\
\hline Alcohol intake & $7(17.5 \%)$ & II (27.5\%) & Ns \\
\hline Hypertension & $28(70 \%)$ & $34(85 \%)$ & Ns \\
\hline Coronary disease & $14(35 \%)$ & $19(47.5 \%)$ & Ns \\
\hline Chronic heart failure & $18(45 \%)$ & $23(57.5 \%)$ & Ns \\
\hline Chronic kidney disease & $4(10 \%)$ & $3(7.5 \%)$ & Ns \\
\hline Cirrhosis & $2(5 \%)$ & $5(12.5 \%)$ & Ns \\
\hline Chronic obstructive pulmonary & $13(32.5 \%)$ & $17(42.5 \%)$ & Ns \\
\hline
\end{tabular}

\section{NDMPs, TNF-A, IL-6, sTREM-I, PCT and} Clinical Parameters in Sepsis or Septic Shock Patients

The plasma NDMPs concentration in the septic shock patients was significantly higher than those in the sepsis patients during the $1^{\text {st }}$ day to the $5^{\text {th }}$ day of ICU admission $(p<0.05)$. There was no significant difference in the plasma levels of NDMPs between sepsis and septic shock patients on the $7^{\text {th }}$ day $(p>0.05)$ (Figure 2A). Compared with the sepsis group, the pro-inflammatory factors (TNF-a, IL-6, sTREM-1), PCT, APACHE II score and MODS score were significantly higher in the septic shock group from the $1^{\text {st }}$ day to the $7^{\text {th }}$ day of ICU admission $(p<0.05)$ (Figure 2B-G). Mechanical ventilation time, ICU LOS and total hospital LOS in the septic shock group were significantly longer than those of the sepsis group $(p<0.05)$ (Figure 2H-J).

\section{Relationship Between Plasma NDMPs} Concentration and Pro-Inflammatory Cytokines and Clinical Outcome Data

The plasma concentration of NDMPs was positively correlated with TNF- $\alpha(\mathrm{r}=0.828, p<0.01)$, IL-6 ( $\mathrm{r}=0.955$, $p<0.001)$, PCT $(\mathrm{r}=0.851, p<0.01)$, sTREM-1 levels $(\mathrm{r}=0.831, \quad p<0.01), \quad$ APACHE II scores $\quad(\mathrm{r}=0.792$, $\mathrm{p}<0.01)$, MODS scores $(\mathrm{r}=0.605, p<0.05)$, mechanical ventilation time $(\mathrm{r}=0.892, p<0.01)$, ICU LOS $(\mathrm{r}=0.309$, $p<0.05)$ and total hospital LOS $(\mathrm{r}=0.933, p<0.001)$ (Figure 3A-I). The correlation coefficient of NDMPs and IL-6 was the greatest among those measured variables.

The plasma NDMP concentration of all patients on the post-ICU admission day 1, 3, 5 and 7 was divided into four groups according to ascending quartile analysis (Quartiles cut-off points of NDMPs concentration $9.7 \times 10^{7} / \mathrm{mL}, 3.55 \times 10^{8} / \mathrm{mL}, 6.4 \times 10^{8} / \mathrm{mL}$ ). The levels of TNF- $\alpha$ and PCT were increased significantly with the increase of NDMPs concentration $(p<0.05)$. The level of IL-6 showed an increasing trend but did not reach to a statistical significance $(p>0.05)$. With the increase of NDMPs concentration, sepsis severity assessment index (APACHE II score, MODS score and mechanical ventilation time, ICU LOS and total hospital LOS) was also increased significantly $(p<0.05)$ (Figure $4 A-D)$.

\section{Comparison of Various Experimental Variables Between Survivals and Non-Survivals}

Various variables including the NDMPs, sTREM-1, TNF- $\alpha$, IL-6, APACHE II score, MODS score, mechanical ventilation time, ICU LOS, total hospital LOS and 28-day mortality between the survival and non-survival group were analyzed. When compared with the survivals, the NDMPs, sTREM-1, TNF- $\alpha$, IL-6, APACHE II score, MODS score were significantly higher in the nonsurvivals from the $1^{\text {st }}$ day to the $7^{\text {th }}$ day of ICU admission $(p<0.05)$ (Figure 5A-G). The 28-day mortality, mechanical ventilation time, ICU LOS, total hospital LOS were significantly higher in the non-survivals than those in the survivals $(p<0.05)$ (Table 2) (Figure $5 \mathrm{H}-\mathrm{J})$. 

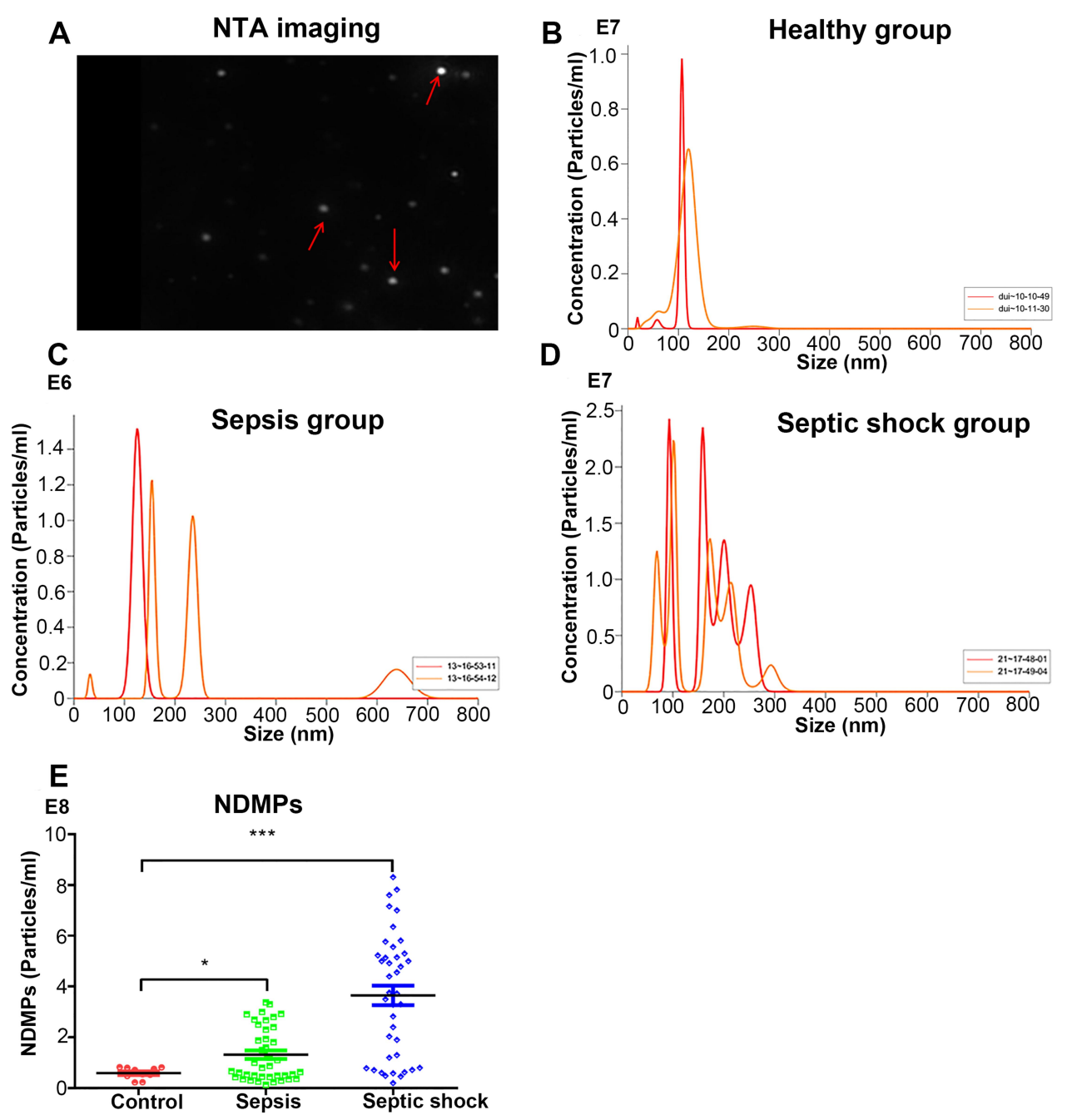

Figure I The plasma micro-particles (NDMPs) in healthy controls, sepsis and septic shock patients. (A) Nanoparticle tracking analysis imaging of NDMPs (red arrow). An example measurement trace of a healthy control group (B), sepsis (C) and septic shock patient (D). (E) Dot plot of plasma NDMPs in healthy individuals, sepsis and septic shock patients. $* P<0.05$ and $* * * P<0.001$.

\section{Predictive Value of Plasma NDMPs Concentration for Mortality}

Survival curves were divided among 4 quartiles based on the plasma NDMPs concentration from low to high for all patients from the $1^{\text {st }}$ day to the $7^{\text {th }}$ day of the ICU admission ranked by ascending quartile method (the quartiles' cut-off points of NDMPs concentration were $9.7 \times 10^{7} / \mathrm{mL}, 3.55 \times 10^{8} / \mathrm{mL}$, and $6.4 \times 10^{8} / \mathrm{mL}$ ). The ability to predict mortality by the scoring system was assessed with receiver operating characteristic (ROC) curve analysis. The mortality was increased significantly with an increase in the plasma NDMPs concentration (Log rank test $P=0.006$ ). When compared with Quartile 1 as a reference, the hazard ratios were 1.746 (0.655-4.653, $p=0.279), 2.66(1.101-6.167, p=0.029)$ and $3.723(1.697-8.165, p=0.002)$ for Quartile 2,
Quartile 3 and Quartile 4, respectively (Figure 6A). The sensitivity and specificity of NDMP levels to predict death with operating characteristic (ROC) curve analysis (Figure 6B) was given the area under the curve (AUC) to be 0.77 . When the NDMPs concentration set to be $3.55 \times 10^{8} / \mathrm{mL}$ as the cut-off point, the sensitivity and specificity were 0.882 and 0.630 , respectively.

\section{Discussion}

Microparticles (MPs), cell membrane-derived particles, play a critical role in both the initiation and development of sepsis. There are a number of studies describing the presence of the endothelial-derived MPs, ${ }^{28-30}$ platelet-derived $\mathrm{MPs},{ }^{29,31,32}$ or leukocyte-derived $\mathrm{MPs}^{29,33}$ in cohorts of patients with sepsis or septic 


\section{Sepsis}
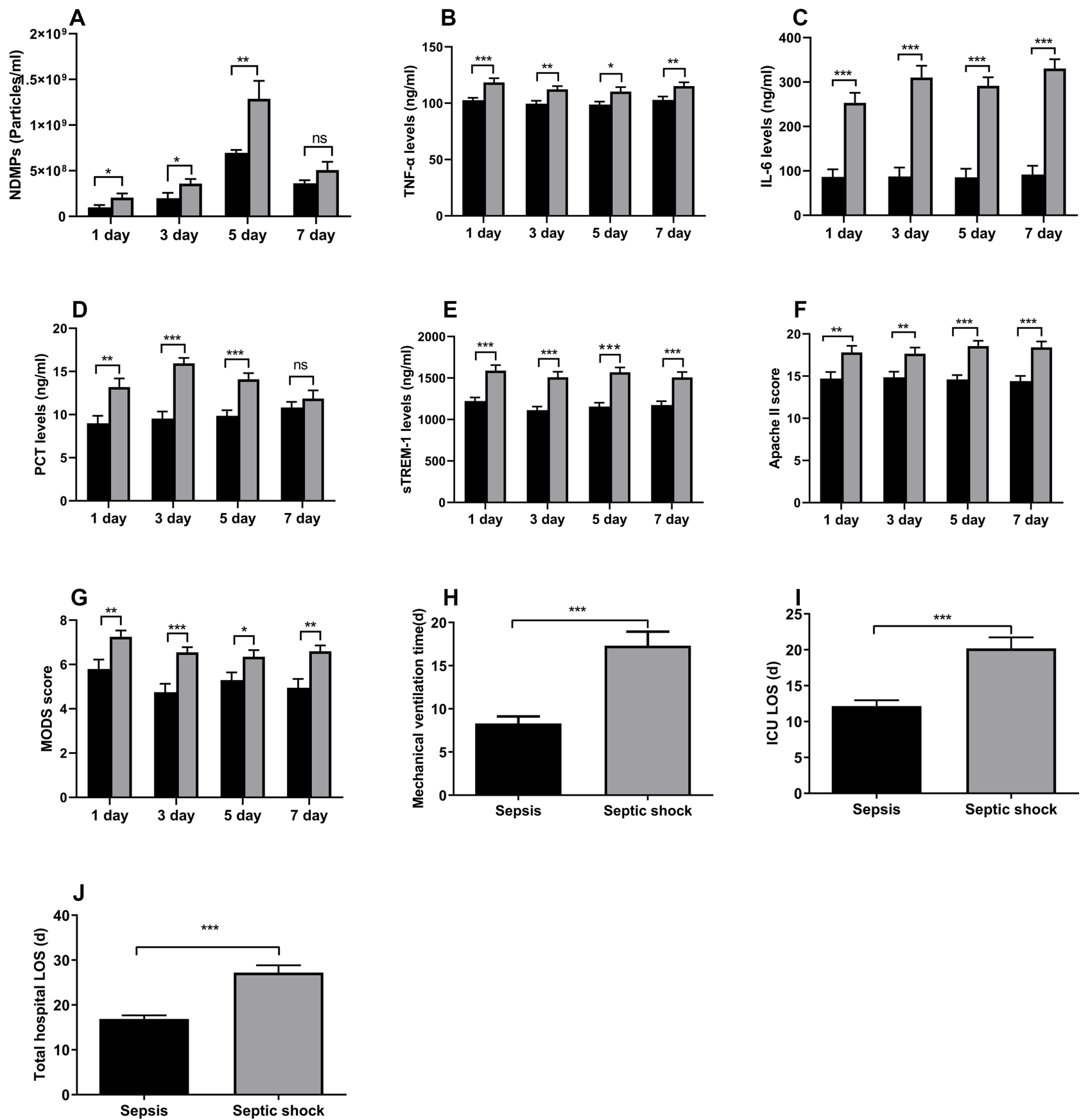

Figure 2 The plasma levels of all measurements and clinical outcomes. NDMPs (A), TNF- $\alpha$ (B), IL-6 (C), PCT (D), sTREM-I (E), APACHE II score (F) and MODS score (G) on days I (40 cases in sepsis group versus 40 cases in septic shock group), 3 (38 versus 36 cases), 5 ( 33 versus 30 cases) and 7 (29 versus 25 cases); mechanical ventilation time $(\mathbf{H})$, ICU LOS $(\mathbf{I})$ and total hospital LOS $(\mathbf{J})$ between sepsis and septic shock groups. Data were mean \pm SD and analyzed by $t$-test. $* p<0.05$, $* * p<0.0 \mathrm{I}, * * * p<0.00 \mathrm{I}$.

shock. Although neutrophils are the most abundant immune cells in blood and the first responders to bacterial infection, the role of neutrophil-derived MPs (NDMPs) in the development of sepsis or septic shock remains undetermined. In the present study, changes in NDMPs during the early phase of sepsis and septic shock were determined and their changes correlated with patients' outcomes were assessed. A significant increase in plasma NDMP concentration in patients with sepsis and septic shock was found 

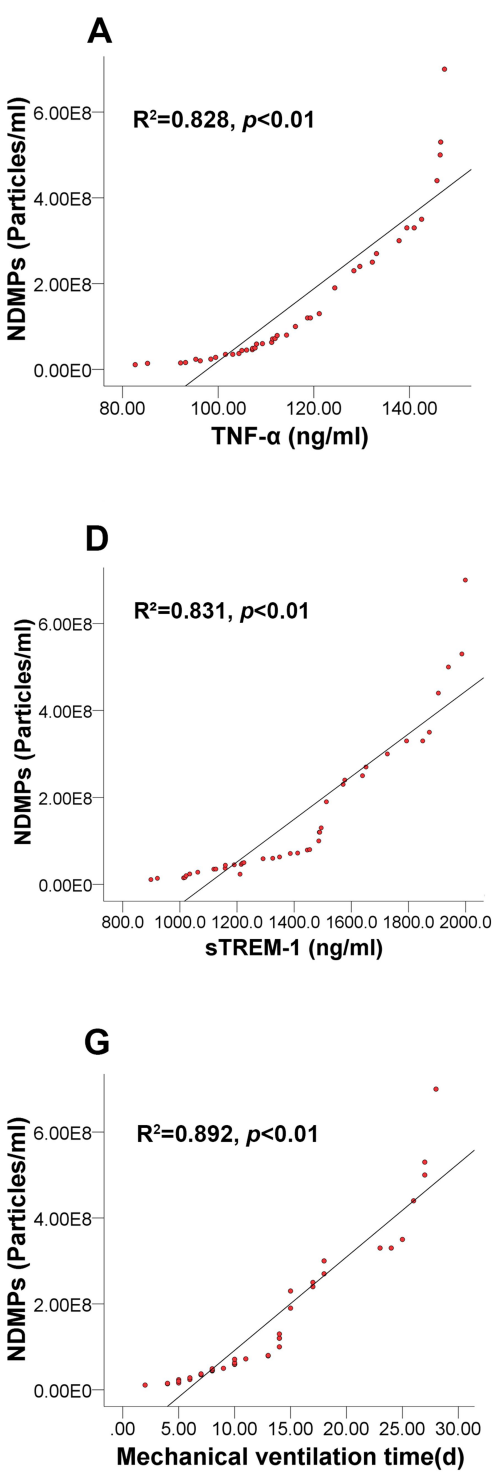

B

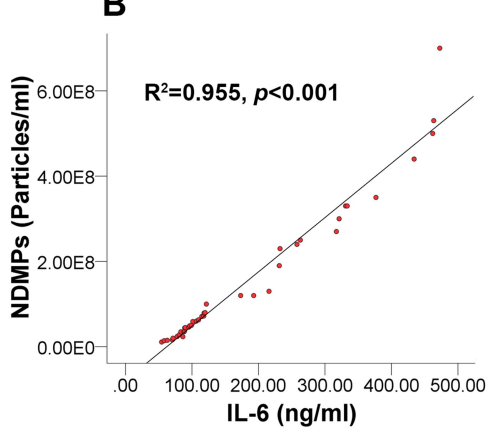

E

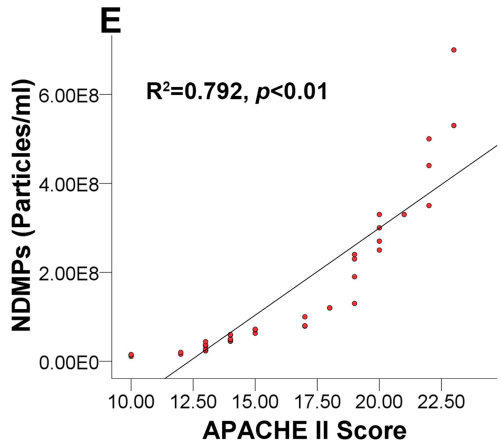

APACHE II Score

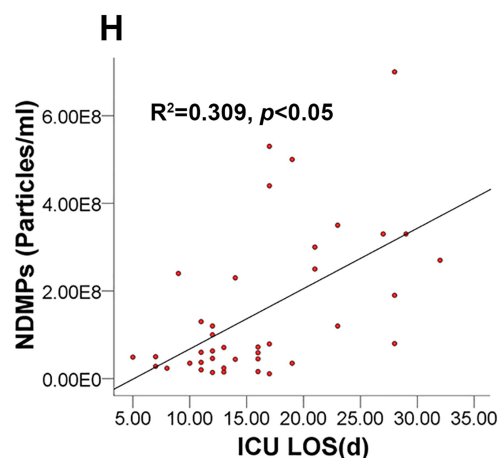

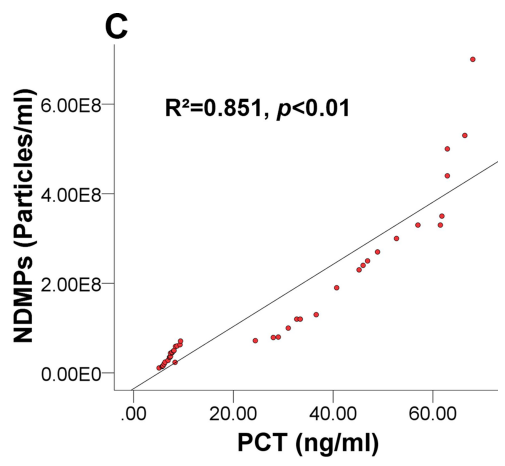
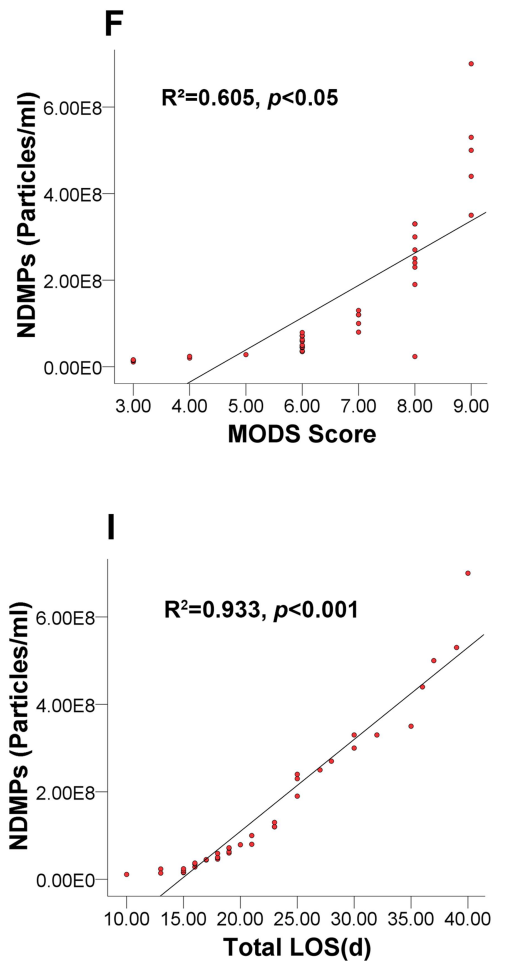

Figure 3 Linear correlations between plasma NDMPs concentration and the levels of all measurements and clinical outcomes. TNF- $\alpha$ (A), IL-6 (B), PCT (C) and sTREM-I (D), Apache II score (E), MODS score (F), mechanical ventilation time $(\mathbf{G})$, and ICU LOS $(\mathbf{H})$, and total hospital LOS (I) in sepsis and septic shock patients.

when compared with the healthy controls; their concentrations in the septic shock patients were even significantly higher than those in the sepsis patients. During the early stage of sepsis, from day 1 to day 5 after ICU admission, levels of NDMPs continuously increased as the sepsis developed, suggesting that neutrophils were activated by microbes and, in turn, produced abundant NDMPs. As sepsis persisted, neutrophils underwent apoptosis and, in turn, generated a further increase in NDMPs. A high level of circulating NDMPs was found to be correlated with poor outcomes in our study.
Furthermore, we found that APACHE II scores, MODS scores, mechanical ventilation time, ICU LOS and total hospital LOS were positively correlated with NDMPs, suggesting that NDMPs were associated with the severity of sepsis. Accumulating evidence indicates that cytokines (ie IL-6, TNF- $\alpha$ ), cell surface markers and soluble receptors (ie sTREM-1) play important roles in sepsis prognosis. ${ }^{34,35}$ Our data also showed that pro-inflammatory mediators such as TNF- $\alpha$, sTREM-1 and IL-6 were significantly positively correlated with NDMPs, and were increased with a parallel increase of plasma NDMPs concentration, suggesting 

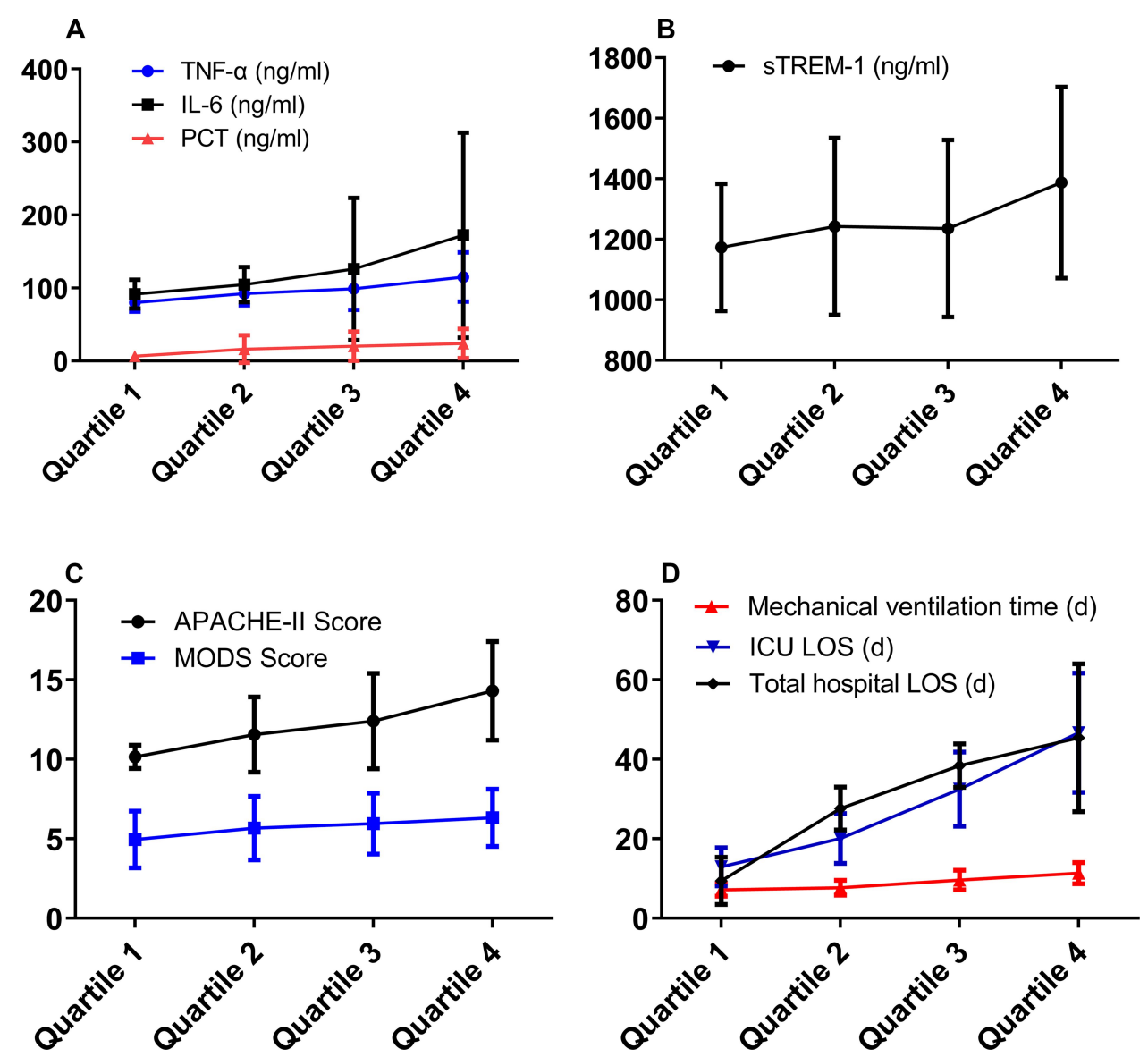

Figure 4 The correlation between plasma NDMPs concentration and mechanical ventilation time, ICU LOS, total hospital LOS, disease severity and inflammatory factors in sepsis and septic shock patients. After arranging the plasma NDMPs concentration of all the patients on days I,3,5,7 by an ascending quartile method (quartiles cutoff points of NDMPs concentration $\left.9.7 \times 10^{7} / \mathrm{mL}, 3.55 \times 10^{8} / \mathrm{mL}, 6.4 \times 10^{8} / \mathrm{mL}\right)$, following the gradual increase of NDMPs concentration, the $p$ values from statistical analysis were: (A) TNF- $\alpha p=0.0045$, IL-6 $p=0.0537$, PCT $p=0.0128$, (B) sTREM-I $p=0.0403$, (C) Apache II Score $p<0.00$ I, MODS Score $p=0.0486$, (D) Mechanical ventilation time $p<0.00$ I, ICU LOS $p<0.00$ I, Total hospital LOS $p<0.00$ I).

that NDMPs likely promoted the release of proinflammatory mediators. NDMPs can be released from activated or apoptotic immune cells by sub-lethal or lethal stimuli. TNF- $\alpha^{36}$ or bacterial byproducts including endotoxin and fMLP $^{10,37}$ are all potent inducers of NDMP generation. Thus, the increase of TNF- $\alpha$, IL-6 and sTREM-1 in our patients may be considered to be mediators to induce the generation of NDMPs. As TNF- $\alpha$ is a cytokine that predominates in the initial stages of sepsis, a murine study determined that activation of either TNF receptor (TNFr1) or TNF receptor 2 (TNFr2) resulted in increased NDMP numbers. ${ }^{36}$ Inhibition of Caspase 8 diminishes NDMPs generated through TNFr1 activation and inhibition of NF- $\mathrm{B}$ abrogates NDMPs generated through activation of both TNFr1 and TNFr2. We conclude that the early production of TNF-during sepsis can increase NDMP numbers through activation of the Caspase 8 pathway or $\mathrm{NF}-\kappa \mathrm{B}$.

In addition, we also found that the plasma NDMP concentration in non-surviving patients was significantly higher than that in surviving patients, and with an increase in plasma NDMP concentration, there was a concomitant increase in sepsis mortality. This may suggest that NDMPs are closely related to the prognosis of sepsis. The ROC curve analysis showed that the value of NDMPs to predict mortality of sepsis patients is considerably high. Previous in vitro studies $^{13}$ have reported that NDMPs are phagocytosed by monocytes which cause amplified activation of the ingesting leukocytes and deactivation of surrounding cells. The activation of these cells likely exacerbates immune suppression. The leukocyte ingestion of NDMPs and apoptosis can lead to fewer immune cells 


\section{$\square$ Survival $\square$ Non-survival}
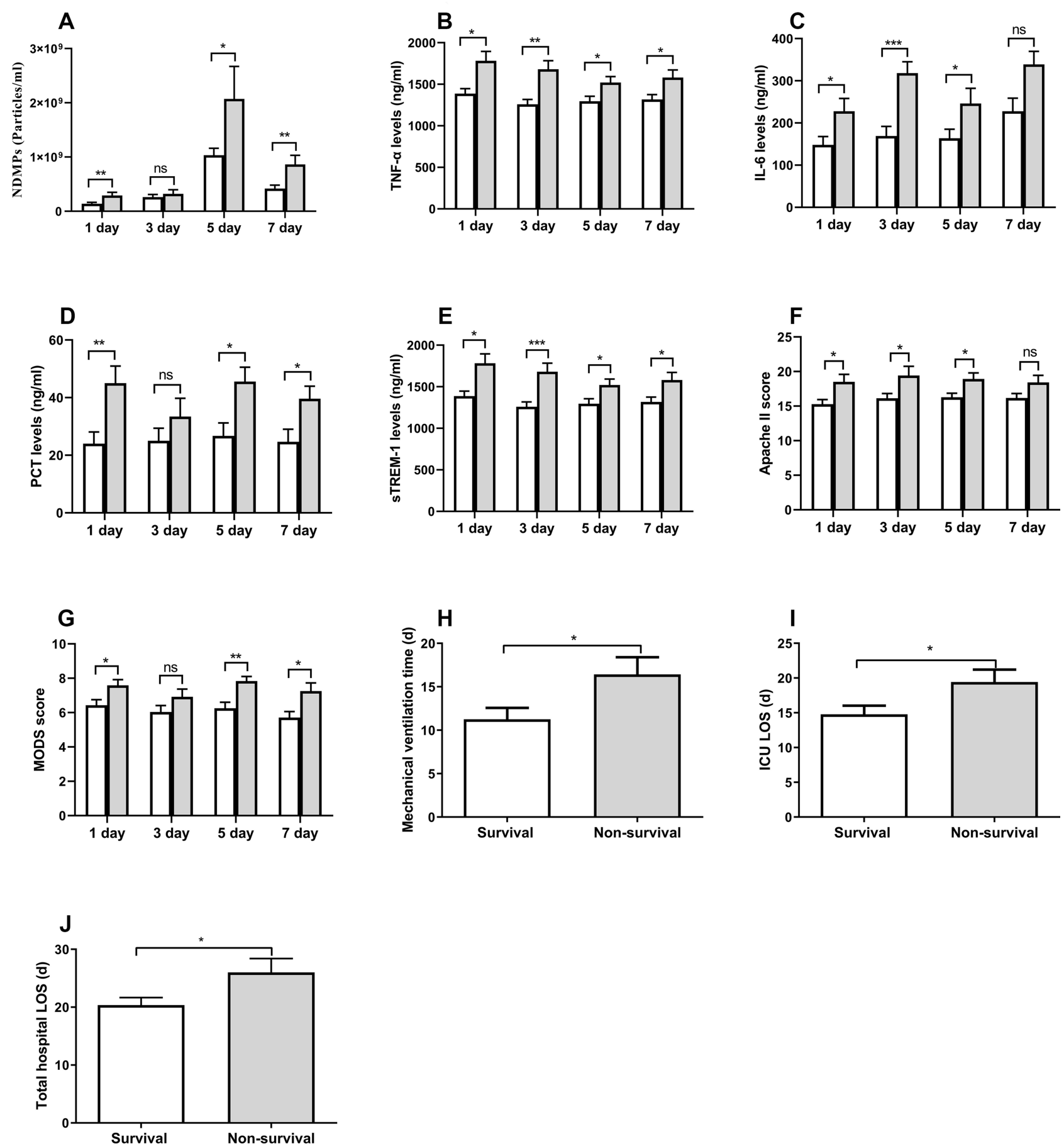

Figure 5 Comparisons between surviving and non-surviving patients of all measurements and clinical outcomes. Plasma concentration of NDMPs (A), TNF- $\alpha$ (B), IL-6 (C), PCT (D), sTREM-I (E), Apache II score (F) and MODS score (G) on days I (54 cases in survival group versus 26 cases in non-survival group), 3 (52 versus 22 cases), 5 (49 versus 17 cases) and 7 (45 versus I4 cases); and mechanical ventilation time (H), and ICU LOS (I) and total hospital LOS (J) in survival and non-survival groups. Data were mean \pm SD and analyzed by $t$-test. $* p<0.05, * * p<0.01$, $* * * p<0.001$ compared with sepsis group.

available for mounting an antimicrobial response. MPs contain phosphatidylserine which is a signal for phagocytosis $^{38}$ and once cells ingested NDMPs, they became activated and their phagocytic ability was increased. It was found that NDMPs are phagocytosed by THP-1 cells and they have a divergent effect on the immune response by activating phagocytic cells and deactivating bystander cells during sepsis in 
Table 2 Clinical Outcomes in Patients with Sepsis or Septic Shock

\begin{tabular}{|l|l|l|l|}
\hline & $\begin{array}{l}\text { Sepsis } \\
\text { Group } \\
(\mathbf{n}=40)\end{array}$ & $\begin{array}{l}\text { Septic Shock } \\
\text { Group } \\
(\mathbf{n = 4 0 )}\end{array}$ & P value \\
\hline $\begin{array}{l}\text { 28-day mortality } \\
\text { Mechanical ventilation }\end{array}$ & $\begin{array}{l}\text { II (27.5\%) } \\
\text { time (day) }\end{array}$ & $\begin{array}{l}15(37.5 \%) \\
17.30 \pm 0.82\end{array}$ & $\begin{array}{l}0.2123 \\
<0.00 \mathrm{I}\end{array}$ \\
$\begin{array}{l}\text { ICU LOS (day) } \\
\text { Total hospitalized LOS } \\
\text { (day) }\end{array}$ & $12.15 \pm 0.8 \mathrm{I}$ & $20.20 \pm 1.54$ & $<0.00 \mathrm{I}$ \\
\hline
\end{tabular}

Abbreviation: LOS, length of stay.

patients. ${ }^{13}$ In terms of cytokine signaling, NDMPs isolated from septic patients have been shown to activate JNK1-specific signaling in endothelial cells, leading to the release of IL- 6 and MCP-1. ${ }^{39}$ In a further study, it was demonstrated that NDMPs caused endothelial cells to generate IL-6 and IL-8 as well as increased expression of adhesion molecules. ${ }^{40}$ A separate study demonstrated that NDMPs bind to endothelial cells via CD18, resulting in increased ICAM-1 expression as well as increased ROS generation by endothelial cells. $^{41}$

NDMPs also caused immune dysfunction in sepsis by blunting the function of neutrophils and macrophages, and subsequent increased mortality in a murine model of sepsis. ${ }^{42}$ Clinically, the exacerbation of immunosuppression represents a significant risk to the morbidity and mortality of patients with septic shock. ${ }^{43}$

All these indicate that NDMPs are likely involved in sepsis development and may be considered novel and rapid biomarkers of the severity of sepsis. Furthermore, NDMPs also influence the pathophysiology of a disease during sepsis. A sustained overabundance of NDMPs can cause an overwhelming acute inflammatory response which would be detrimental to all vital organs during sepsis development.

The diagnosis of sepsis and septic shock at the early stage is essential and any delay in initiating proper treatments is associated with poor prognosis. ${ }^{44}$ Therefore, detecting plasma NDMPs immediately after ICU admission would be helpful for better understanding the severity and prognosis of sepsis and promoting proper treatments. However, it is acknowledged that all measurements in this study and clinical outcomes reported herein are only associations and are within an observational nature. The cause-effect relationship among NDMPs, cytokines and patients' outcome is unknown. In addition, the relatively small sample size of this study is another limitation. All these factors combined mean that caution with regards to the interpretation of this data is needed, though further study in this area is certainly warranted.

\section{Conclusions}

In conclusion, we found that NDMPs together with pro-inflammatory mediators were significantly increased in sepsis and even further increased in septic shock patients. Those increases were associated with the severity of sepsis. Plasma NDMPs may be considered to be a prognostic biomarker of sepsis severity and mortality but their role in sepsis prognosis warrants further study.
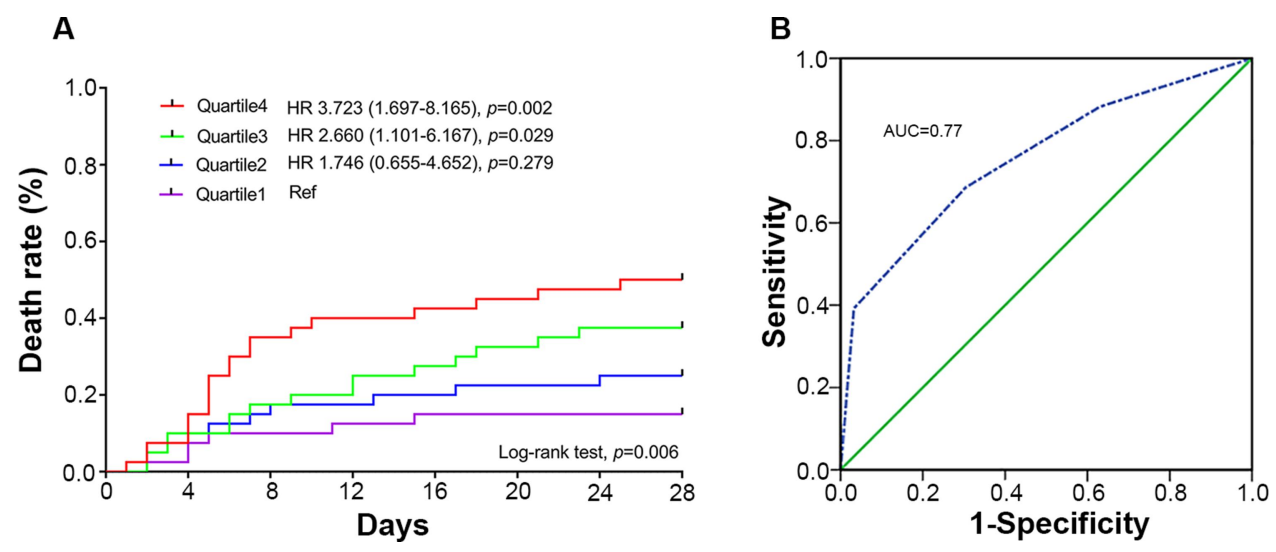

Figure 6 NDMPs vs survival prediction. (A) Patient death among 4 quartiles based on the plasma NDMPs concentration from low to high of all the patients on the postadmission day 1,3,5 and 7 arranged by the ascending quartile method (Quartiles cut-off points of NDMPs concentration $9.7 \times 10^{7} / \mathrm{mL}, 3.55 \times 10^{8} / \mathrm{mL}, 6.4 \times 10^{8} / \mathrm{mL}$ ), Log-rank (Mantel-Cox) test, $p=0.006 \mathrm{I}$. (B) ROC curve of the sensitivity and specificity of NDMP levels to predict death in patients with sepsis and septic shock. 


\section{Abbreviations}

MP, microparticles; NDMPs, neutrophil derived microparticles; TNF- $\alpha$, tumour necrosis factor; IL-6, interleukin-6; STREM-1, soluble triggering receptor expressed on myeloid cells-1; PCT, procalcitonin; APACHE II Score, Acute Physiology and Chronic Health Evaluation II Score; MODS, multiple organ dysfunction syndrome; LOS, ICU length of stay.

\section{Data Sharing Statement}

All data are available in the manuscript or upon request to the authors.

\section{Ethics Approval and Informed Consent}

All procedures followed were in accordance with the ethical standards of the responsible committee on human experimentation (institutional and national) and with the Helsinki Declaration of 1975 , as revised in 2008. Informed consent was obtained from all patients or their caring relatives for being included in the study.

\section{Acknowledgments}

The authors thank Dr. Yiming Fan for critical comments. This study was supported by Zhanjiang Key Laboratory of Organ Injury and Protection and Translational Medicine, Zhanjiang, Guangdong, China.

\section{Funding}

This study was supported by grant NO. 81974298 from the National Natural Science Foundation of China (Beijing, China).

\section{Disclosure}

The authors report no competing interests.

\section{References}

1. Timar CI, Lorincz AM, Csepanyi-Komi R, et al. Antibacterial effect of microvesicles released from human neutrophilic granulocytes. Blood. 2013;121(3):510-518. doi:10.1182/blood-2012-05-431114

2. Rossaint JKK, Skupski J. Directed transport of neutrophil-derived extracellular vesicles enables platelet-mediated innate immune response. Nature commun. 2017;7:13464.

3. Boulanger CMAN, Tedgui A. Circulating microparticles: a potential prognostic marker for atherosclerotic vascular disease. Hypertension. 2006;48(2):180-186. doi:10.1161/01.HYP.0000231507.00962.b5

4. Morel O, Toti F, Hugel B, et al. Procoagulant microparticles: disrupting the vascular homeostasis equation? Arterioscler Thromb Vasc Biol. 2006;26(12):2594-2604. doi:10.1161/01.ATV.0000246775.14471.26
5. Martinez MC, Tesse A, Zobairi F, Andriantsitohaina R. Shed membrane microparticles from circulating and vascular cells in regulating vascular function. Am J Physiol Heart Circ Physiol. 2005;288(3): H1004-9. doi:10.1152/ajpheart.00842.2004

6. Distler J, Huber LC, Huber LC, Kalden JR, Gay S, Distler O. Microparticles as regulators of inflammation: novel players of cellular crosstalk in the rheumatic diseases. Arthritis Rheum. 2005;52 (11):3337-3348. doi:10.1002/art.21350

7. Hickey MJ, Kubes P. Intravascular immunity: the host-pathogen encounter in blood vessels. Nat Rev Immunol. 2009;9(5):364-375. doi: $10.1038 /$ nri2532

8. Nathan C. Neutrophils and immunity: challenges and opportunities. Nat Rev Immunol. 2006;6(3):173-182. doi:10.1038/nri1785

9. Kolaczkowska E, Kubes P. Neutrophil recruitment and function in health and inflammation. Nat Rev Immunol. 2013;13(3):159-175. doi:10.1038/nri3399

10. Gasser O, Schifferli JA. Activated polymorphonuclear neutrophils disseminate anti-inflammatory microparticles by ectocytosis. Blood. 2004;104(8):2543-2548. doi:10.1182/blood-2004-01-0361

11. Butin-Israeli V, Houser MC, Feng M, et al. Deposition of microparticles by neutrophils onto inflamed epithelium: a new mechanism to disrupt epithelial intercellular adhesions and promote transepithelial migration. FASEB J. 2016;30(12):4007-4020. doi:10.1096/fj.20160 0734R

12. Y J SS Y, Kim JK. Neutrophil-derived extracellular vesicles: proinflammatory trails and anti-inflammatory microvesicles. bioRxiv. 2019;583435.

13. Prakash PS, Caldwell CC, Lentsch AB, Pritts TA, Robinson BR. Human microparticles generated during sepsis in patients with critical illness are neutrophil-derived and modulate the immune response. J Trauma Acute Care Surg. 2012;73(2):401-6; discussion 406-7. doi:10.1097/TA.0b013e31825a776d

14. Chang PS, Absood A, Linderman JJ, Omann GM. Magnetic bead isolation of neutrophil plasma membranes and quantification of membrane-associated guanine nucleotide binding proteins. Anal Biochem. 2004;325(2):175-184. doi:10.1016/j.ab.2003.10.039

15. Gautam A, Dixit S, Embers M, et al. Different patterns of expression and of IL-10 modulation of inflammatory mediators from macrophages of Lyme disease-resistant and -susceptible mice. PLoS One. 2012;7(9):e43860. doi:10.1371/journal.pone.0043860

16. Novotny AR, Reim D, Assfalg V, et al. Mixed antagonist response and sepsis severity-dependent dysbalance of pro- and anti-inflammatory responses at the onset of postoperative sepsis. Immunobiology. 2012;217(6):616-621. doi:10.1016/j.imbio.2011.10.019

17. Zhao GJ, Lu ZQ, Tang LM, et al. Curcumin inhibits suppressive capacity of naturally occurring $\mathrm{CD} 4+\mathrm{CD} 25+$ regulatory $\mathrm{T}$ cells in mice in vitro. Int Immunopharmacol. 2012;14(1):99-106. doi:10.10 16/j.intimp.2012.06.016

18. Wang P, Ba ZF, Chaudry IH. Mechanism of hepatocellular dysfunction during early sepsis. Key role of increased gene expression and release of proinflammatory cytokines tumor necrosis factor and interleukin-6. Arch Surg. 1997;132(4):364-9;discussion 369-70. doi:10.1001/archsurg.1997.01430280038005

19. Xu XJ, Tang YM, Liao C, et al. Inflammatory cytokine measurement quickly discriminates gram-negative from gram-positive bacteremia in pediatric hematology/oncology patients with septic shock. Intensive Care Med. 2013;39(2):319-326. doi:10.1007/s00134-012$2752-4$

20. Arora S, Singh P, Singh PM, Trikha A. Procalcitonin levels in survivors and nonsurvivors of sepsis: systematic review and meta-analysis. Shock. 2015;43(3):212-221. doi:10.1097/SHK.000000 0000000305

21. Masson S, Caironi P, Spanuth E, et al. Presepsin (soluble CD14 subtype) and procalcitonin levels for mortality prediction in sepsis: data from the Albumin Italian Outcome Sepsis trial. Crit Care. 2014;18(1):R6. doi:10.1186/cc13183 
22. Zhang J, She D, Feng D, Jia Y, Xie L. Dynamic changes of serum soluble triggering receptor expressed on myeloid cells-1 (sTREM-1) reflect sepsis severity and can predict prognosis: a prospective study. BMC Infect Dis. 2011;11:53. doi:10.1186/1471-2334-11-53

23. Pitanga TN, de Aragao Franca L, Rocha VC, et al. Neutrophilderived microparticles induce myeloperoxidase-mediated damage of vascular endothelial cells. BMC Cell Biol. 2014;15:21. doi:10.1186/ 1471-2121-15-21

24. Rhodes A, Evans LE, Alhazzani W, et al. Surviving sepsis campaign: international guidelines for management of sepsis and septic shock: 2016. Crit Care Med. 2017;45(3):486-552. doi:10.1097/CCM.000 0000000002255

25. Singer M, Deutschman CS, Seymour CW, et al. The Third International Consensus Definitions for Sepsis and Septic Shock (Sepsis-3). JAMA. 2016;315(8):801-810. doi:10.1001/jama.2016. 0287

26. Wang J, Zhong Y, Ma X, et al. Analyses of endothelial cells and endothelial progenitor cells released microvesicles by using microbead and Q-dot based nanoparticle tracking analysis. Sci Rep. 2016;6:24679. doi:10.1038/srep24679

27. Dragovic RAGC, Brooks AS. Sizing and phenotyping of cellular vesicles using nanoparticle tracking analysis. Nanomedicine. 2011;7 (6):780-788. doi:10.1016/j.nano.2011.04.003

28. Liu Y, Zhang R, Qu H, Wu J, Li L, Tang Y. Endothelial microparticles activate endothelial cells to facilitate the inflammatory response. Mol Med Rep. 2017;15(3):1291-1296. doi:10.3892/mmr.2017.6113

29. Boscolo A, Campello E, Bertini D, et al. Levels of circulating microparticles in septic shock and sepsis-related complications: a case-control study. Minerva Anestesiol. 2019;85(6):625-634. doi:10.23736/S0375-9393.18.12782-9

30. Combes V, Simon AC, Grau GE, et al. In vitro generation of endothelial microparticles and possible prothrombotic activity in patients with lupus anticoagulant. J Clin Invest. 1999;104 (1):93-102. doi:10.1172/JCI4985

31. Lentz BR. Exposure of platelet membrane phosphatidylserine regulates blood coagulation. Prog Lipid Res. 2003;42(5):423-438. doi:10.1016/s0163-7827(03)00025-0

32. Siddiqui FA, Desai H, Amirkhosravi A, Amaya M, Francis JL. The presence and release of tissue factor from human platelets. Platelets. 2002;13(4):247-253. doi:10.1080/09537100220146398

33. Lehner GF, Harler U, Haller VM, et al. Characterization of microvesicles in septic shock using high-sensitivity flow cytometry. Shock. 2016;46(4):373-381. doi:10.1097/SHK.0000000000000657
34. Herzum I, Renz H. Inflammatory markers in SIRS, sepsis and septic shock. Curr Med Chem. 2008;15(6):581-587. doi:10.2174/09298670 8783769704

35. Lam HS, Ng PC. Biochemical markers of neonatal sepsis. Pathology. 2008;40(2):141-148. doi:10.1080/00313020701813735

36. Johnson BL 3rd, Goetzman HS, Prakash PS, Caldwell CC. Mechanisms underlying mouse TNF-alpha stimulated neutrophil derived microparticle generation. Biochem Biophys Res Commun. 2013;437(4):591-596. doi:10.1016/j.bbrc.2013.06.118

37. Pluskota E, Woody NM, Szpak D, et al. Expression, activation, and function of integrin alphaMbeta2 (Mac-1) on neutrophil-derived microparticles. Blood. 2008;112(6):2327-2335. doi:10.1182/blood2007-12-127183

38. Eken C, Sadallah S, Martin PJ, Treves S, Schifferli JA. Ectosomes of polymorphonuclear neutrophils activate multiple signaling pathways in macrophages. Immunobiology. 2013;218(3):382-392. doi:10.1016/ j.imbio.2012.05.021

39. Mesri M, Altieri DC. Leukocyte microparticles stimulate endothelial cell cytokine release and tissue factor induction in a JNK1 signaling pathway. J Biol Chem. 1999;274(33):23111-23118. doi:10.1074/jbc.2 74.33.23111

40. Mesri M, Altieri DC. Endothelial cell activation by leukocyte microparticles. J Immunol. 1998;161(8):4382-4387.

41. Hong Y, Eleftheriou D, Hussain AA, et al. Anti-neutrophil cytoplasmic antibodies stimulate release of neutrophil microparticles. 2012;23 (1):49-62. doi:10.1681/ASN.2011030298

42. Johnson BL 3rd, Midura EF, Prakash PS, et al. Neutrophil derived microparticles increase mortality and the counter-inflammatory response in a murine model of sepsis. Biochim Biophys Acta Mol Basis Dis. 2017;1863(10 Pt B):2554-2563. doi:10.1016/j.bbadis.20 17.01.012

43. Wheeler AP, Bernard GR. Treating patients with severe sepsis. $N$ Engl J Med. 1999;340(3):207-214. doi:10.1056/NEJM19990121 3400307

44. Kumar A, Roberts D, Wood KE, et al. Duration of hypotension before initiation of effective antimicrobial therapy is the critical determinant of survival in human septic shock. Crit Care Med. 2006;34(6):1589-1596. doi:10.1097/01.CCM.0000217961.75225.E9
Journal of Inflammation Research

\section{Publish your work in this journal}

The Journal of Inflammation Research is an international, peerreviewed open-access journal that welcomes laboratory and clinical findings on the molecular basis, cell biology and pharmacology of inflammation including original research, reviews, symposium reports, hypothesis formation and commentaries on: acute/chronic inflammation; mediators of inflammation; cellular processes; molecular mechanisms; pharmacology and novel anti-inflammatory drugs; clinical conditions involving inflammation. The manuscript management system is completely online and includes a very quick and fair peerreview system. Visit http://www.dovepress.com/testimonials.php to read real quotes from published authors. 